\title{
Texture Evolution and Ultrafine Grain Formation in Cross-Cryo-Rolled Zircaloy-2
}

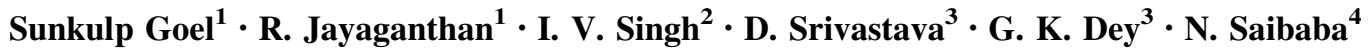

Received: 2 November 2014/Revised: 3 March 2015/Published online: 8 April 2015

(C) The Chinese Society for Metals and Springer-Verlag Berlin Heidelberg 2015

\begin{abstract}
The texture and mechanical properties of cross-rolled zircaloy- 2 at 77 and $300 \mathrm{~K}$ were investigated. Crossrolling at $77 \mathrm{~K}$ was performed to impart different thickness reductions of $25 \%$ and $50 \%$, while at $300 \mathrm{~K}$ with $25 \%, 50 \%$, $75 \%$ and $85 \%$ reductions to the sample. EBSD analysis of deformed sample showed that near-basal orientation is not deformed completely after 50\% rolling reduction. The activation of prismatic silp, $\{11 \overline{2} 2\}$ contraction twin and $\{10 \overline{1} 2\}$ extension twin were evident from the deformed microstructure at $77 \mathrm{~K}$. The propensity for activation of basal slip $<a>$ at $77 \mathrm{~K}$ was also observed. The deformation of the sample at $300 \mathrm{~K}$ occurs by prismatic, basal $<a>$ and pyramidal $<c+a>$ slips, which were predicted by pole figures. After annealing, the tensile strengths (735 and $710 \mathrm{MPa}$ ) are almost the same for $50 \%$ cryo-cross-rolled and room-temperature cross-rolled zircaloy- 2 with almost $2.7 \%$ difference in their ductility. KAM analysis of the deformed samples was made to estimate the stored strain energy and dislocation density. Annealing of deformed sample at $673 \mathrm{~K}$ for 30 min results in recrystallization, which leads to the formation of ultrafine grains.
\end{abstract}

\section{KEY WORDS: Cross-rolling; EBSD; TEM; Ultrafine grains; Texture}

\section{Introduction}

Zircaloy-2 is widely used in nuclear reactor due to the good corrosion resistance, mechanical properties and low neutron cross section [1-3]. The elastic, plastic and thermal properties of $\mathrm{Zr}$ alloys are anisotropic due to the hcp

Available online at http://link.springer.com/journal/40195

R. Jayaganthan

rjayafmt@iitr.ernet.in

1 Department of Metallurgical and Materials Engineering, Centre of Nanotechnology, IIT Roorkee, Roorkee 247667, India

2 Department of Mechanical and Industrial Engineering, IIT Roorkee, Roorkee 247667, India

3 Materials Science Division, Bhabha Atomic Research Center, Mumbai 40085, India

4 Nuclear Fuel Complex Limited, Hyderabad 501301, India structure of it [4]. The texture development in zircaloy-2 alloy during thermomechanical processing strongly influences its anisotropic deformation characteristics [5, 6]. Primary mode of deformation in zircaloy- 2 occurs by twinning and slip as reported in the literatures [7-10]. Prismatic slip $\{10 \overline{1} 0\}\langle 1 \overline{2} 10\rangle$ in $\mathrm{Zr}$ alloys has been observed at all temperatures [11, 12]. Pyramidal and basal slips in hcp alloys result in small homogeneous strain without change in volume [13]. However, prismatic and $\langle c+a>$ pyramidal slips could activate at low strain easily, while basal $\langle a\rangle$ slip activates when prismatic becomes strain hardened at high strain in zirconium alloys $[14,15]$. The deformation in zirconium occurs mostly by twinning at low temperature [15-17]. In extension loading along $c$ axis, $\{10 \overline{1} 2\}\langle\overline{1} 011\rangle$ twin gets activated and $\{11 \overline{2} 1\}\langle\overline{11} 26\rangle$ twins get activated less often. During contraction loading along $c$ axis, $\{11 \overline{2} 2\}\langle\overline{11} 23\rangle$ twins are active, while $\{10 \overline{1} 1\}\langle\overline{1} 012\rangle$ twins are also seen [10]. At $77 \mathrm{~K}$, deformation is governed mainly by prismatic slip, extension twinning $\{10 \overline{1} 2\}\langle\overline{1} 011\rangle$, 
$\{11 \overline{2} 1\}\langle\overline{11} 26\rangle$ and contraction twinning $\{11 \overline{2} 2\}\langle\overline{11} 23\rangle$ as reported in the literatures $[8,10,16,17]$.

Schmid factor is an important parameter, a link between the applied stress and the critically resolved shear stress on a specific slip system. It is a relation between the applied loading directions and the slip direction of a crystal, given by the relation $\cos \varphi \cos \alpha$, in which $\varphi$ is the angle between slip direction and loading axis, while $\alpha$ is the angle between slip plane normal and loading axis [18]. The slip system with the highest Schmid factor would operate first. During plastic deformation, reorientation of the crystal occurs, leading to texture development in the alloy, which affects the mechanical behavior of the material and thus promotes anisotropy in property [19]. In addition to texture, grain size influences the strength of the zircaloy- 2 in accordance with the Hall-Petch effect [20, 21]. The individual grains behave independently within polycrystalline materials during plastic deformation as reported by Cox and Sopwith [22]. This causes difference in the mechanical properties predicted by using theoretical and experimental results [22]. During deformation, each crystal behavior depends on the neighboring crystal, and a Taylor factor $(M)$ was derived relating the applied stress and CRSS. This factor is widely used to predict the texture formation due to polycrystalline deformation [23, 24], which is defined as

$M=\frac{\sigma_{\mathrm{y}}}{\tau_{0}}$,

where $\sigma_{\mathrm{y}}$ represents yield strength of the sample and $\tau_{0}$ represents CRSS of the activated slip system.

The stored strain energy inside low carbon steel upon plastic deformation depends on the crystal orientation, which results in recrystallization texture as reported in the literature [25]. The stored strain energy of deformed materials is estimated by using the techniques such as calorimetric and diffraction [26, 27]. Diffraction method is further divided into X-rays line broadening and orientation distribution function $[28,29]$. The relation between stored strain energy and Taylor factor has shown that the stored energy increases with increase in Taylor factor with some exceptions [30, 31]. To our knowledge, there is no report on effect of cross-rolling on texture, stored strain energy, microstructure and mechanical properties of zircaloy- 2 . Therefore, the present work was focused to study the texture, microstructure and mechanical properties of zircaloy2 processed by cross-rolling at room temperature and liquid nitrogen temperature. The Schmidt and Taylor factors of the deformed alloy were estimated to substantiate the formation of texture and stored strain energy, respectively.

\section{Experimental Procedure}

Rolled zircaloy-2 sheet with $4 \mathrm{~mm}$ thickness was obtained from nuclear fuel complex, Hyderabad, India, and its chemical composition is given in Table 1. Samples with dimension of $5 \mathrm{~cm} \times 3 \mathrm{~cm}$ were cut and solid solution heat treated at $800{ }^{\circ} \mathrm{C}$ in argon environment followed by quenching in mercury. Samples after solution treatment were surface grounded and washed in methanol. The cleaned samples were then cross-rolled at room temperature and cryo-temperature by changing the orientation by $90^{\circ}$ in every pass as shown in Fig. 1 . The samples were dipped in liquid nitrogen for $10 \mathrm{~min}$ before each pass so as to attain cryo-temperature inside the samples. Liquid nitrogen was continuously poured over the rollers to attain cryo-temperature in rollers. Room temperature was attained by dipping the samples in water after every pass. $\mathrm{MoSi}_{2}$ is used to reduce friction losses. A true strain of 0.04 was given in every pass. With the reduction in thickness of the sample, the rolling reduction was reduced to ensure constant true strain induced into the sample. Baby rolling mill with a speed of $8 \mathrm{r} / \mathrm{min}$ has been used to carry out cross-rolling, and the roller diameter is $110 \mathrm{~mm}$.

Various characterizations such as tensile test, transmission electron microscopy (TEM), electron back scattered diffraction (EBSD) and $\mathrm{X}$ ray diffraction (XRD) were performed to understand the microstructural and mechanical behavior of rolled zircaloy-2. Tensile tests were performed with ASTM: E8 substandard size specimen with a gauge length of $16 \mathrm{~mm}$ with a strain rate of $5 \times 10^{-4} \mathrm{~s}^{-1}$. The samples for TEM investigation were prepared by using twin jet at a voltage of $20 \mathrm{~V}$ at $-48{ }^{\circ} \mathrm{C}$ using 20:80 perchloric acid and methanol solution. TEM was performed by using FEI Tecnai-20 machine $(200 \mathrm{kV})$. Electron back scatter diffraction (EBSD) was carried out by using FEI Quanta 200 FEG-SEM/EBSD with a step size of $0.1 \mu \mathrm{m}$. EBSD analysis was performed by TSL OIM software package. Texture XRD of the samples was carried out by Pan analytical MRD system instrument using $\mathrm{Cu} K_{\alpha}$ radiation with a step size of $0.0017^{\circ}$ and an integration time of $400 \mathrm{~s}$.

Table 1 Chemical composition of Zircaloy-2

\begin{tabular}{llllll}
\hline Element & Tin $(\mathrm{Sn})$ & Iron $(\mathrm{Fe})$ & Chromium $(\mathrm{Cr})$ & Nickel (Ni) & Nitrogen $(\mathrm{N})$ \\
\hline $\mathrm{Wt}$ & $1.3-1.6$ & $0.07-0.20$ & $0.05-0.16$ & $0.03-0.08$ & 0.006 \\
\hline
\end{tabular}




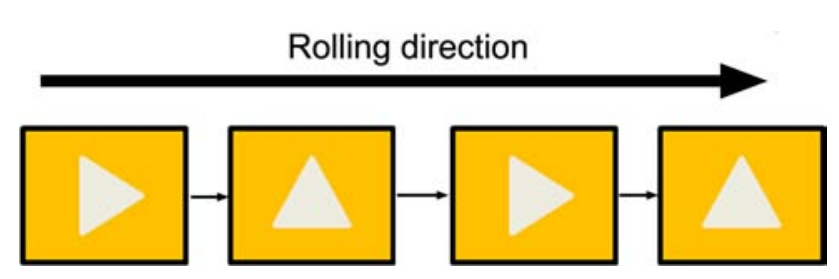

Fig. 1 Schematic diagram showing the cross-rolling method

\section{Results and Discussion}

\subsection{TEM Observation}

Initial microstructure with an average grain size of $10 \mu \mathrm{m}$ was obtained after quenching zircaloy-2 in mercury. Prior to mercury quenching, the alloy was firstly heat treated at $800{ }^{\circ} \mathrm{C}$, so that $\alpha$-structured matrix can be obtained, as reported in earlier works [20, 21]. The TEM micrograph of 25\% cryo-cross-rolled (CCR) sample is shown in Fig. 2a. Dislocation cells with dislocation inside can be seen after $25 \%$ reduction in the alloy. At cryo-temperature, dislocations could accumulate easily due to suppression of dynamic recovery. The dislocation cells of very small size are formed at cryo-temperature. On the other hand, $25 \%$ roomtemperature cross-rolled (RTCR) depicts the formation of dislocation cells of large size as compared to cryo-temperature, as shown in Fig. 2b. Fewer dislocations are trapped inside them after $25 \%$ reduction due to dynamic recovery at room temperature. During rolling, some of the energy is converted into heat, which is also responsible for dynamic recovery inside the grain. The dislocation cells are low-angle grain boundaries, which are also called incidental dislocation boundaries (IDBs). Since the true strain is very less, geometrically necessary boundaries (GNBs) are not observed in both the samples. When the strain is increased to $50 \%$, dislocation density inside the cells has increased.

With the change in strain path during each rolling pass, plastic flow instability occurs due to destabilization of substructure caused by the deformation in the new rolling direction. It affects the dislocation configuration and local barriers of dislocation network [32]. In 50\% CCR sample, ultrafine grains formed in some locations due to change in orientation, which is evidenced by the macroscopic plastic flow of the dislocation cells and stacking faults, as shown in the inset of Fig. 2c. In 50\% RTCR zircaloy-2, dislocation cells with dislocations trapped inside were observed, as shown in Fig. 2d. Ultrafine grains are also formed in the alloy due to macroscopic plastic flow of dislocation cells. Insets of Fig. $2 \mathrm{c}$ and d show the selected area diffraction (SAD) patterns, which prove grain formation, while the elongated pattern shows the variation in lattice parameter. Further reduction up to $85 \%$ is possible only in roomtemperature cross-rolled sample as high CRSS of cryorolled alloy causes cracking after $50 \%$ thickness reduction.

Annealing the rolled samples at $673 \mathrm{~K}$ for 30 min leads to rearrangement of dislocation, which facilitates the nucleation of grains inside the crystals, as shown in Fig. 3. The internal stress inside the crystals increases with increasing plastic strain, and it is developed locally due to the hcp structure and orientations of crystals. Annealing at low temperature provides the driving force for the nucleation of deformed grains in these places. The grains that are already formed due to dynamic recrystallization transformed into dislocation free grains upon annealing treatment. In $25 \%$ CCR and 25\% RTCR samples, grains with low-angle grain boundaries inside the white squares are observed in Fig. 3a, b. The formation of ultrafine grains has increased with increasing strain followed by annealing as evident from Fig. 3.

\subsection{Mechanical Properties}

Mechanical properties of the CCR and RTCR samples are listed in Table 2 . With every pass during cross-rolling, the strain path and loading direction over the sample get changed due to which more defects are generated. CCR sample showed substantial improvement in strength
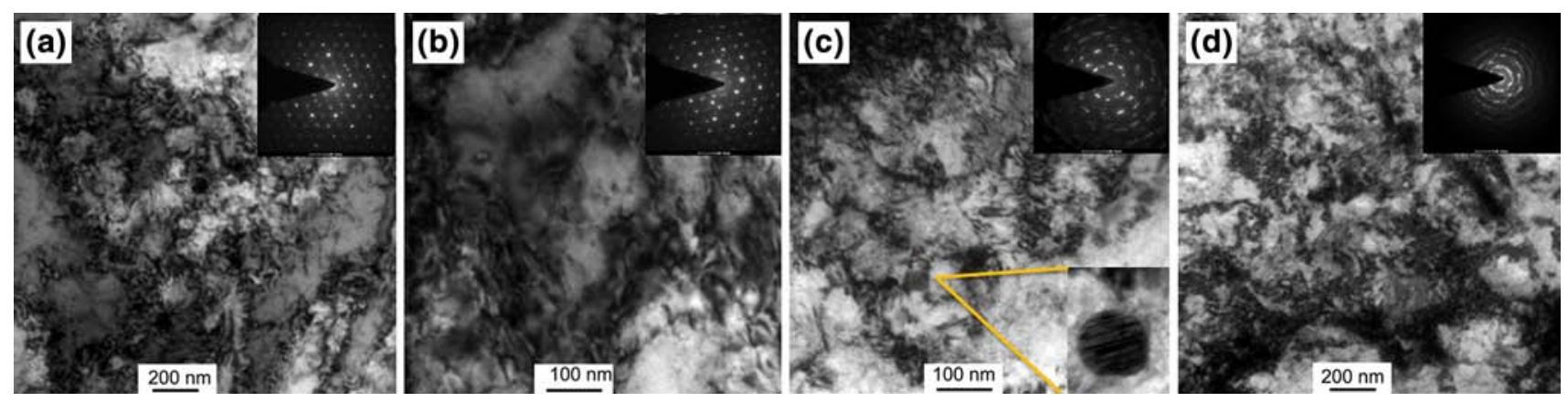

Fig. 2 TEM images of cryo-cross-rolled (CCR) and room-temperature cross-rolled (RTCR) zircaloy-2: a 25\% CCR; b 25\% RTCR; c 50\% CCR; d $50 \%$ RTCR 

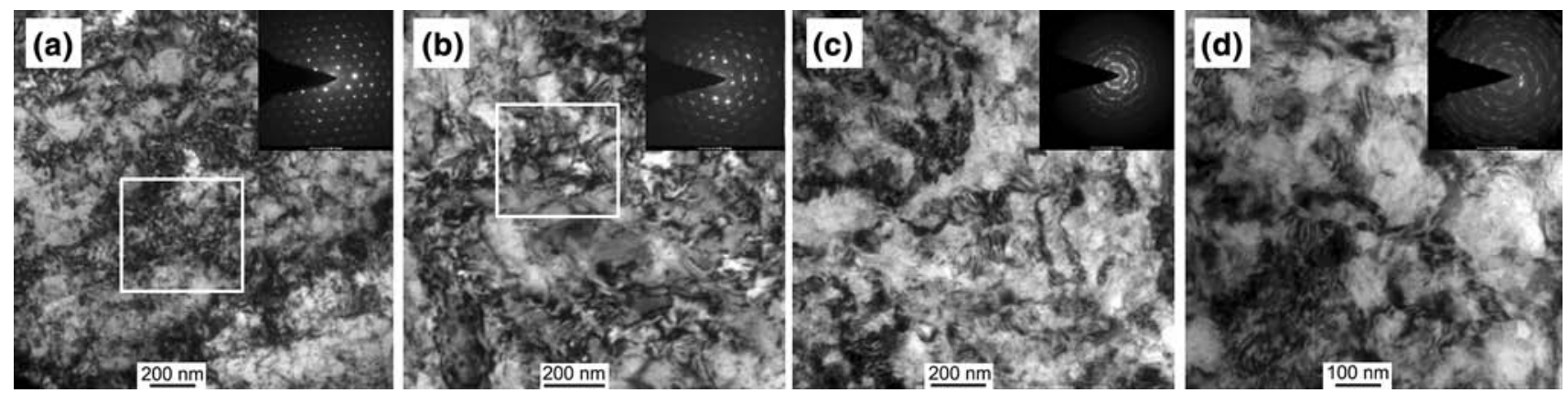

Fig. 3 TEM images of cryo-cross-rolled (CCR) and room-temperature cross-rolled (RTCR) zircaloy-2 after annealing at $400{ }^{\circ} \mathrm{C}$ for 30 min: a $25 \%$ CCR; b $25 \%$ RTCR; c 50\% CCR; d 50\% RTCR

Table 2 Mechanical properties of Zircaloy-2 after cross rolling

\begin{tabular}{lllll}
\hline & Hardness (HV) & Tensile strength (MPa) & Yield strength (MPa) & \% Elongation at break \\
\hline Mercury quenched & 182 & 499 & 331 & 25 \\
$25 \%$ RTCR & 211 & 693 & 632 & 13.3 \\
$50 \%$ RTCR & 233 & 727 & 684 & 10.45 \\
$25 \%$ CCR & 215 & 734 & 698 & 10.2 \\
$50 \%$ CCR & 237 & 786 & 753 & 8.7 \\
$25 \%$ RTCR Annld & 211 & 628 & 592 & 16.2 \\
$50 \%$ RTCR Annld & 233 & 710 & 634 & 12.5 \\
$25 \%$ CCR Annld & 215 & 659 & 621 & 12.8 \\
$50 \%$ CCR Annld & 237 & 735 & 703 & 9.8 \\
\hline
\end{tabular}

compared to RTCR alloy due to its higher dislocation density achieved through suppression of dynamic recovery. The change in strain path during each pass also induces plastic strain inside the crystals, and therefore, only for $50 \%$ reduction in thickness, the formation of ultrafine and nanograins has occurred inside the matrix, which are not observed for normal cryo-rolled alloy, as reported in earlier works [20, 21]. Large dislocation densities as well as ultrafine grains are responsible for strain hardening of the alloy. At cryo-temperature, thickness reduction beyond $50 \%$ was not achieved due to fracture of samples owing to high CRSS. Tensile strength of 50\% CCR alloy was found to be $786 \mathrm{MPa}$ with $8.7 \%$ ductility, while the RTCR alloy showed strength of $727 \mathrm{MPa}$ with $10.45 \%$ ductility at break. The strength and ductility of room-temperaturerolled zircaloy- 2 after $85 \%$ reduction, reported in our earlier work, showed $13 \%$ and $36 \%$ lesser, respectively, as compared to CCR sample. The tensile strength and ductility of RTR alloy were less by $6.0 \%$ and $47.0 \%$, respectively, as compared to RTCR alloy in the present work. The increase in ductility may be due to multimodal grain structure and movement of preexisting dislocation at higher stress $[12,33,34]$.

Annealing at $673 \mathrm{~K}$ for $30 \mathrm{~min}$ results in nucleation and grain growth in deformed zircaloy-2, thus forming ultrafine and nanocrystalline grains. Due to hep structure, internal stress developed during deformation is not uniformly distributed, which causes anisotropic grain growth leading to the formation of multimodal grain structure in the alloy. Annealing at low temperature improves the ductility without the drop in strength. The improvement in ductility of 50\% CCR zircaloy-2 was $9.8 \%$, while $12.5 \%$ of RTCR zircaloy-2 after annealing. The tensile strength of CCR and RTCR samples was found to be 735 and $710 \mathrm{MPa}$, respectively. The $25 \%$ RTCR alloy upon annealing showed a ductility of $16.2 \%$ with tensile strength of $628 \mathrm{MPa}$. The improvement in ductility is $62.9 \%$ of $25 \%$ RTCR zircaloy-2, and it is $85 \%$ of RTR annealed zircaloy-2 whose tensile strength almost equal, which was reported earlier [21].

\subsection{EBSD and KAM Analysis}

It is observed from Fig. 4a that after 25\% CCR, grains with near-basal orientation, in red color, are left non-deformed as they are elastically harder [35]. Low-angle misorientations are increasing, which are dislocation cells and tangles as seen from TEM results. The misorientation chart in Fig. $4 \mathrm{c}$ shows that the low-angle grain boundaries are increasing with increase in reduction. Grains with some 

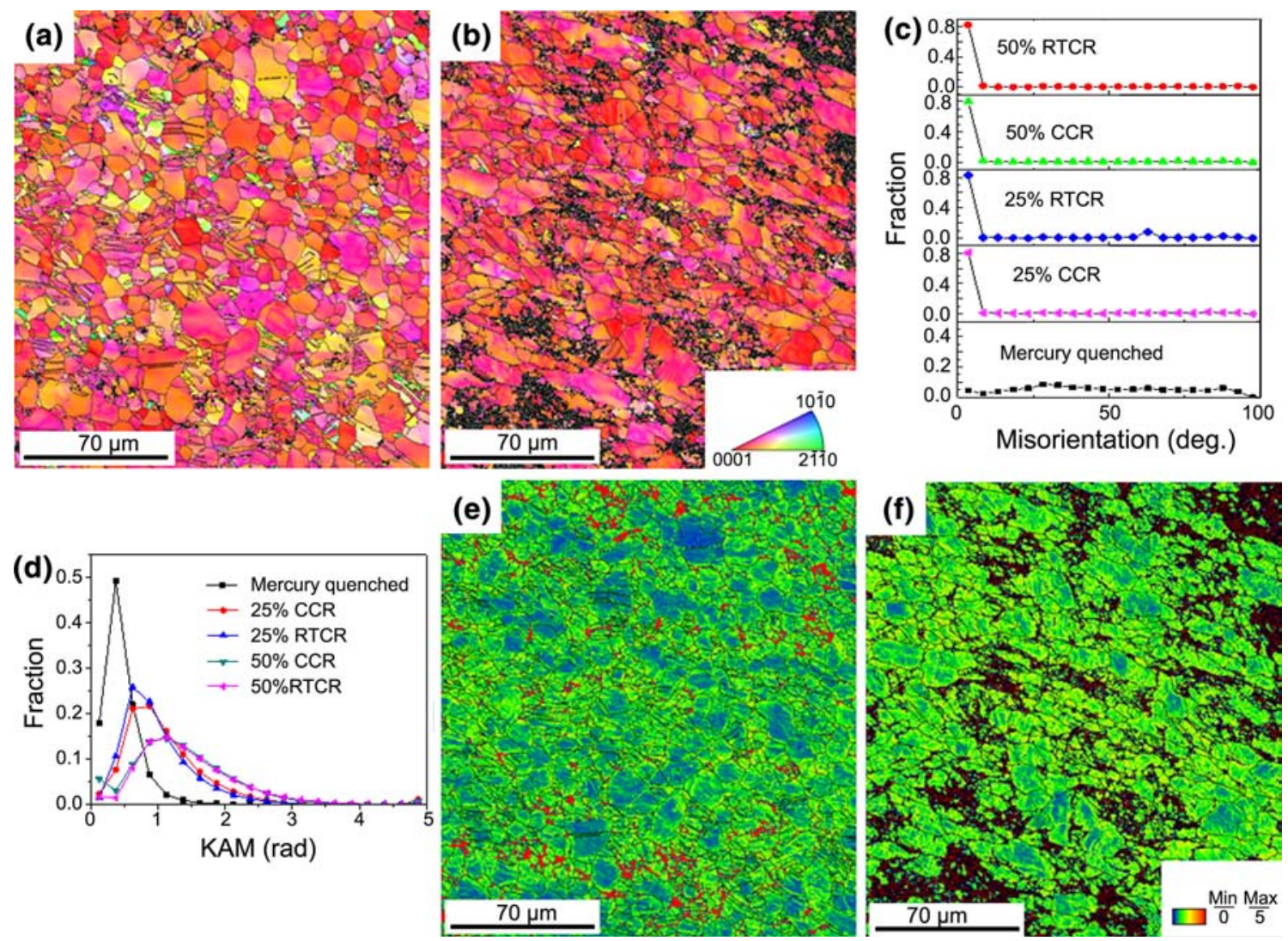

Fig. 4 EBSD images of 25\% a and 50\% b CCR zircaloy-2; average misorientation $\mathbf{c}$ and KAM d of CCR and RTCR zircaloy-2; KAM images of $25 \%$ e, $50 \%$ f CCR zircaloy- 2

partitions in $25 \%$ CCR are seen from Fig. 4a show orientation of about $20^{\circ}$, which may be evidence of $\{11 \overline{2} 1\}$ extension twinning, and it has disappeared after misorientation due to activation of slip inside the crystal. In the $50 \%$ CCR sample, only grains with near-basal orientation are left undeformed because they are non-fragmenting and elastically harder (Fig. 4b). However, due to change in strain during each pass, dislocations are formed inside these basal grains also, which can be seen from Kernel average misorientation (KAM) (Fig. 4f). CCR and RTCR samples (25\% and 50\%) show high and almost same fraction of low-angle grain boundaries (Fig. 4c), which also proves the effect of change in strain path resulting in more strain hardening.

Kernel average misorientation (KAM) is defined as the average misorientation of the particular point with all its neighbors having misorientation exceeding $5^{\circ}$ is excluded [18]. KAM generally signifies the dislocations and stored energy inside the crystals, and it depends on the step size. The step size of $0.1 \mu \mathrm{m}$ is taken for analyzing the EBSD results in the present work. The red region in the KAM shows very highly deformed regions, whose values are excluded from the graph (Fig. 4d) as such a high misorientation near $5^{\circ}$ on those places shows varying orientation with low $\mathrm{CI}$ index, leading to high value. KAM of $25 \%$ CCR sample (Fig. 4e) shows that most of the grains are with large dislocation density inside them. However, the KAM image of 50\% CCR sample (Fig. 4f) shows the presence of dislocations inside these near-basal non-fragmenting grains, due to change in strain path during rolling. It enables for improving the strength, hardness and ductility. Comparison of both 50\% CCR and RTCR samples shows that there is no major difference in KAM and misorientation (Fig. 4c, d) except little variation in their mechanical properties. Large KAM value can be seen near the grain boundary and in some grains, in $25 \%$ CCR sample. The high KAM value near the grain boundary shows that these grains should have hard orientation. In $50 \%$ CCR sample, almost all the grains with harder orientation show high KAM value, which reflects the effect of change in strain path.

\subsection{Texture}

$\{0002\}$ and $\{01 \overline{1} 2\}$ pole figure (Fig. 5a) of mercuryquenched zircaloy- 2 shows a fully recrystallized structure 

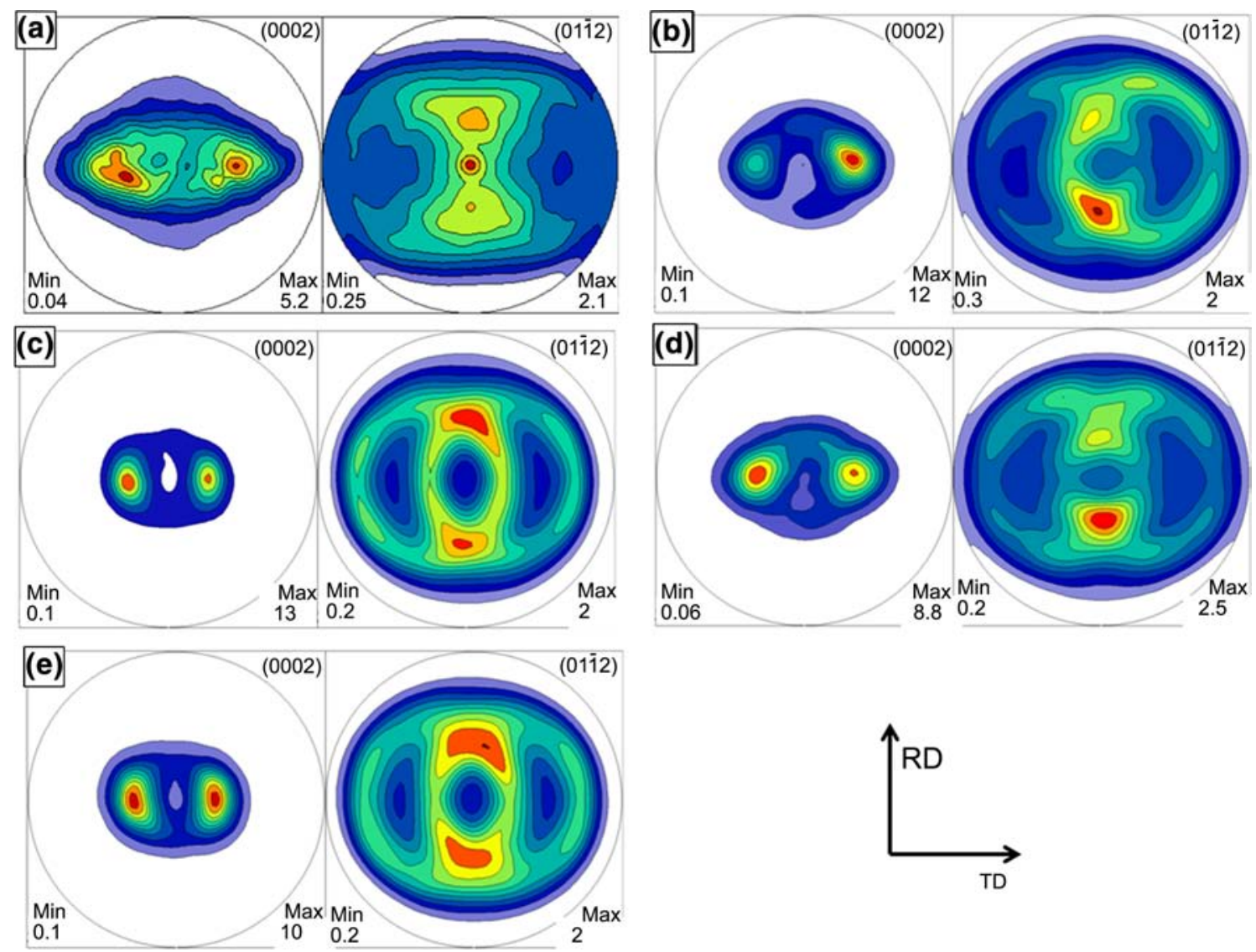

Fig. $5\{0002\}$ and $\{01 \overline{1} 2\}$ pole figure images of zircaloy-2: a mercury quenched; b $25 \%$ CCR; c $50 \%$ CCR; d $25 \%$ CCR after annealing at $400{ }^{\circ} \mathrm{C}$ for $30 \mathrm{~min}$; e $50 \%$ CCR after annealing at $400{ }^{\circ} \mathrm{C}$ for $30 \mathrm{~min}$

with texture toward transverse direction, which are in tandem with the EBSD results in Refs. [20, 21]. After $25 \% \mathrm{CCR},\{0002\}$ poles are aligned $30^{\circ}$ toward the rolling direction and $\{01 \overline{1} 2\}$ poles are shifted toward the transverse direction. They spread toward the rolling direction, as seen from Fig. 5b. The intensity of 25\% CCR sample is 12 , which shows that deformation in CCR sample occurred mostly through twinning. The mercuryquenched zircaloy-2 has shown the texture toward basal orientation. Thus, in CCR sample, apart from $\{10 \overline{1} 2\}$ extension twinning, $\{11 \overline{2} 2\}$ contraction twinning is extensive, as evident from EBSD image [8, 17]. Also, some traces of $\{11 \overline{2} 1\}$ tensile twins are also seen in EBSD (Fig. 4a) [8]. Since there is a change in strain path at each reduction during rolling, deformation due to activation of prismatic slip might have also taken place. $\{0002\}$ and $\{01 \overline{1} 2\}$ pole figure (Fig. 5c) of $50 \%$ CCR sample shows that deformation occurred through primary $\{11 \overline{2} 2\}$ contraction twinning along with secondary $\{10 \overline{1} 2\}$ extension twinning [8]. Basal grains with misorientation inside them can be seen (Fig. 4b), showing activation of slip at cryo-temperature. Prismatic slip might have taken place inside these grains. Further, activation of $\{0002\}$ basal $\langle a\rangle$ slip is also seen from Schmid and Taylor factor analysis discussed in the following section [36]. The intensity of 50\% CCR zircaloy-2 has increased to 13 , showing that destabilization of substructure is minimal and showing texture hardening, which results in large fraction of grains having orientation near basal due to which further reduction causes crack formation leading to failure of materials.

In $25 \%$ RTCR sample, the $\{0002\}$ poles are toward rolling direction, but they are expanded slightly toward the transverse direction, as shown in Fig. 6a. The texture is almost the same in both CCR and RTCR samples except the weak texture observed in $25 \%$ RTCR condition, as its intensity is 7.9. Hence, deformation occurred through both slip and twinning. Initial texture is toward basal showing the activation of $\{10 \overline{1} 0\}$ prismatic slip, and it is dominant in all the grains. While $\{0002\}$ basal $<a\rangle$ slip occurs in non-basal grains, $\{11 \overline{23}\}$ pyramidal $<c+a>$ slip in basal grains might have taken place $[32,37] .\{0002\}$ and $\{01 \overline{1} 2\}$ pole figure of 50\% RTCR zircaloy-2 shows that these poles are spreading toward the rolling direction in both 


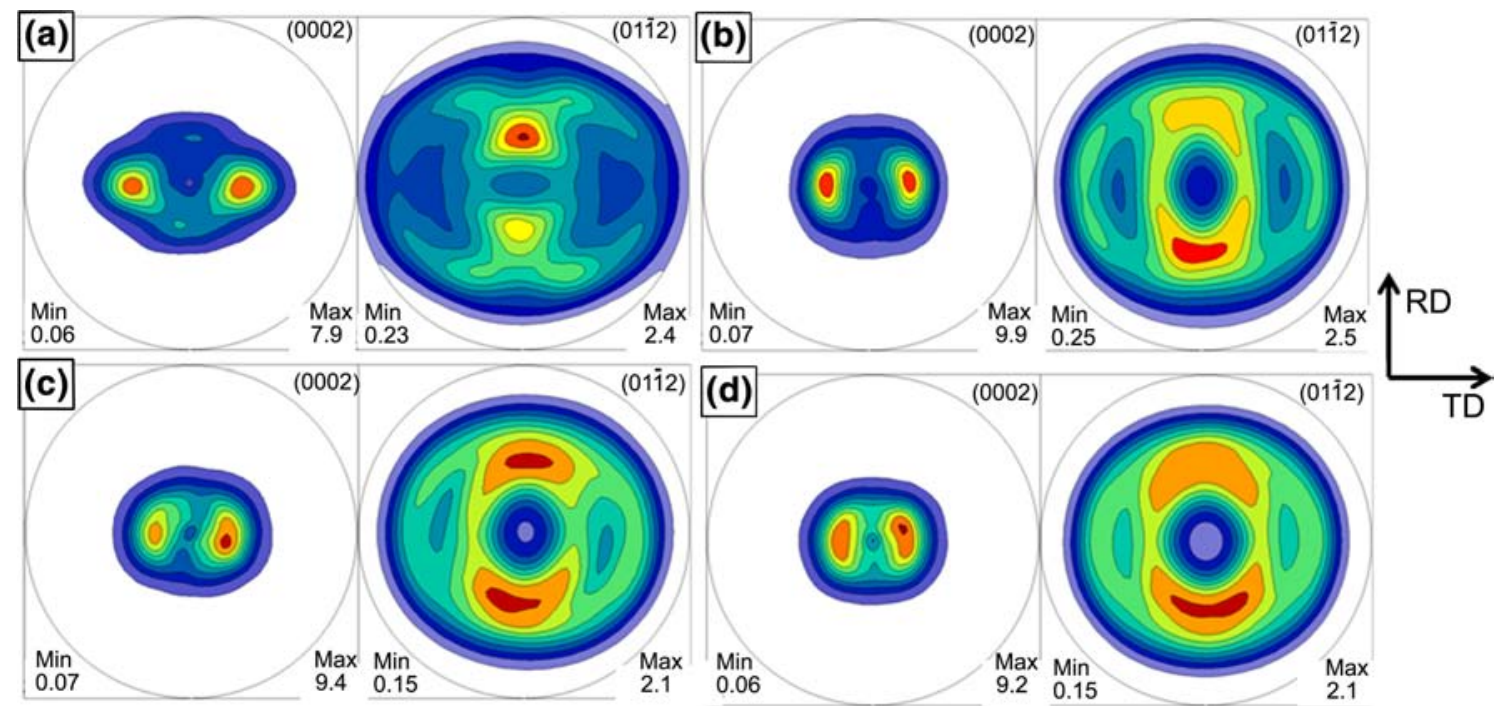

Fig. $6\{0002\}$ and $\{01 \overline{1} 2\}$ pole figure images of $25 \%$ a, $50 \%$ b, $75 \%$ c, $85 \%$ d RTCR zircaloy- 2

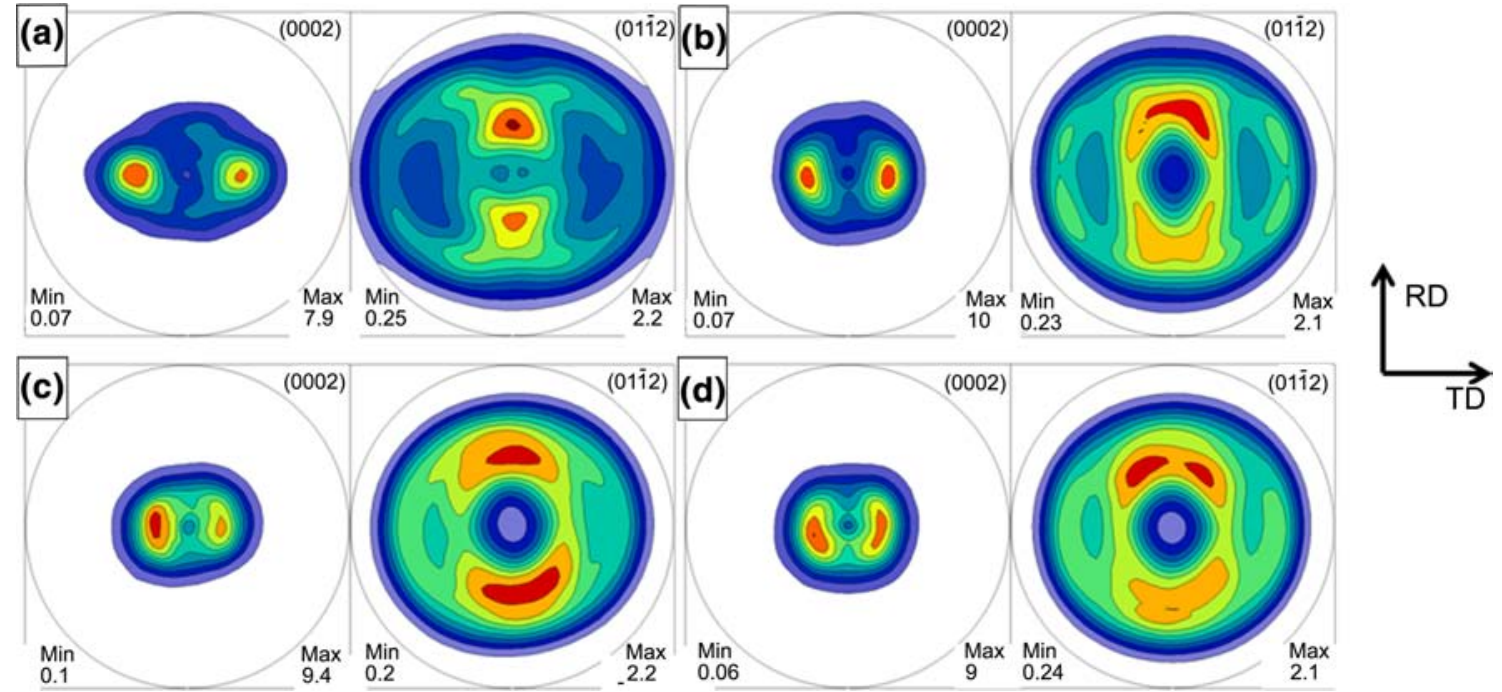

Fig. $7\{0002\}$ and $\{01 \overline{1} 2\}$ pole figure images of $25 \%$ a, $50 \% \mathbf{b}, 75 \% \mathbf{c}, 85 \%$ d RTCR zircaloy- 2 after annealing at $400{ }^{\circ} \mathrm{C}$ for $30 \mathrm{~min}$

conditions (Fig. 6b), which may be due to the change in strain path by cross-rolling. Since most crystals are basal oriented, prismatic, basal $\langle a\rangle$ and pyramidal $\langle c+a\rangle$ slips might have been activated [32], causing deformation to occur mostly by slip up to $50 \%$ reduction. On further reduction in RTCR zircaloy-2 (Fig. 6c, d), the basal poles are inclined due to which pyramidal slip activity has increased and because of change in strain path, basal and prismatic activities were also observed [8, 32].

Annealing causes recrystallization as well as stress relieving to the deformed grains. The grains thus reorient themselves and prismatic plane aligns toward the transverse direction. Here, due to change in strain path, the prismatic planes get inclined in both the rolling directions.
The effect of annealing on $25 \%$ and $50 \%$ CCR samples can be seen from Fig. 5d, e. It clearly shows the weakening of basal texture as well as spreading of $\{0002\}$ basal grains. There is no effect on the intensity after annealing on 25-85\% RTCR samples as seen from Fig. 7.

\subsection{Schmid Factor and Taylor Factor}

Deformation of the crystal occurs in the plane, where RSS is greater than CRSS value. The Taylor's theory is based on the assumption that plastic deformation is caused entirely by dislocation slip and deformation in the grains is almost identical to macroscopic deformation [18]. The Taylor 
factor is the energy required to deform the crystallite in the required orientation of the active combination of slip systems [23]. Therefore, it relates the yield strength of the material to its crystal orientation or texture. The Taylor factor $M$ is defined as

$M=\sum_{\mathrm{s}} \frac{\mathrm{d} \gamma^{\mathrm{s}}}{\varepsilon_{i j}}$

where $\mathrm{d} \gamma^{\mathrm{s}}$ is the CRSS of a particular slip (s) in a given grain and $\varepsilon_{i j}$ is plastic strain applied externally $[18,24]$. Here, it is understood that $M$ depends on the lattice orientation of the crystal. If the value of $M$ is large (near to 4.0), the large amount of slip is required to accommodate large plastic deformation, while low $M$ value indicates that more slip sets are available. Higher $M$ value leads to higher hardening rate of a material [38].

During deformation, slip direction moves toward loading axis, while slip plane normal moves away from loading axis. Higher the Schmid factor shows a high probability of that slip. The Schmid factor is applicable for single-crystal system, and on comparing with Taylor factor, it can be applied to polycrystalline materials also. Schmid factor and Taylor factor can be calculated by defining the loading direction and deformation mechanism with CRSS values. The various CRSS values taken to calculate the Schmid and Taylor

Table 3 Critically resolved shear stress (CRSS) values of slip system at room temperature and cryo-temperature

\begin{tabular}{lll}
\hline Slip & CRSS at $300 \mathrm{~K}$ & CRSS at $77 \mathrm{~K}$ \\
\hline Prism $<a>$ slip & 0.1 & 0.220 \\
Basal $<a>$ slip & 0.16 & 0.260 \\
Pyramidal $<c+a>$ slip & 0.320 & \\
\hline
\end{tabular}

factors are shown in Table 3. The Schmid factor and Taylor factor for $25 \%$ CCR with $\{0002\}$ basal slip can be seen from Fig. 8. It can be seen that majority of grains have both large Schmid and Taylor factors. These grains during deformation have the least probability to activate basal slip due to high Taylor factor. Hence, the basal slip gets strain hardened very fast. Some grains are seen whose Taylor factor is low ( $\ll 2.4)$, but whose Schmid factor is high $(\gg 0.35)$, which are marked by circles in the image. The activation of basal slip will take place in these grains upon further increasing reduction [36]. It has been found that the fraction of grains with low Taylor factor and high Schmid factor are much more in $25 \%$ RTCR sample as compared to $25 \%$ CCR sample. The curves in Fig. 9a and b show Taylor factor and Schmid factor of basal slip of CCR and RTCR samples up to $50 \%$ reduction. It can be seen that $25 \%$ CCR sample, and $25 \%$ and 50\% RTCR samples have low average Taylor factor while high Schmid factor. Mercury quenched and 50\% CCR sample has low Taylor factor as well as low Schmid factor due to which basal slip will not take place in them on further deformation. The Taylor factor and Schmid factor for basal slip of 50\% CCR zircaloy-2 can be seen from Fig. 9c, d. Schmid factor is high for prism slip as it is always active, which has not been shown. The CRSS value for pyramidal slip at cryo-temperature is very high, and therefore, it is not active at low strain.

\subsection{Dislocation Density and Stored Strain Energy}

KAM is average value of misorientation within $5^{\circ}$ with nearby grains, which is used to find out the dislocation density and low-angle grain boundary in the material. During deformation, low-angle tilt and twist boundary consisting of screw dislocations and non-screw dislocations
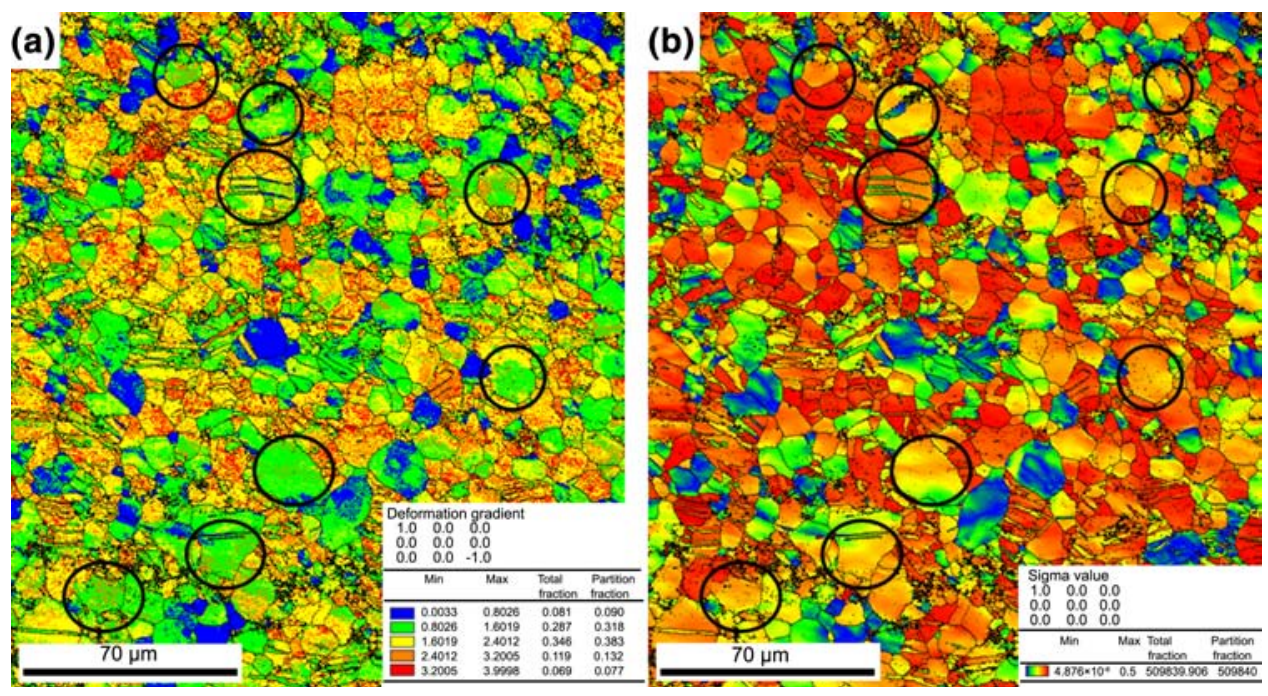

Fig. 8 Taylor factor a, Schmid factor b image of 25\% CCR zircaloy-2 

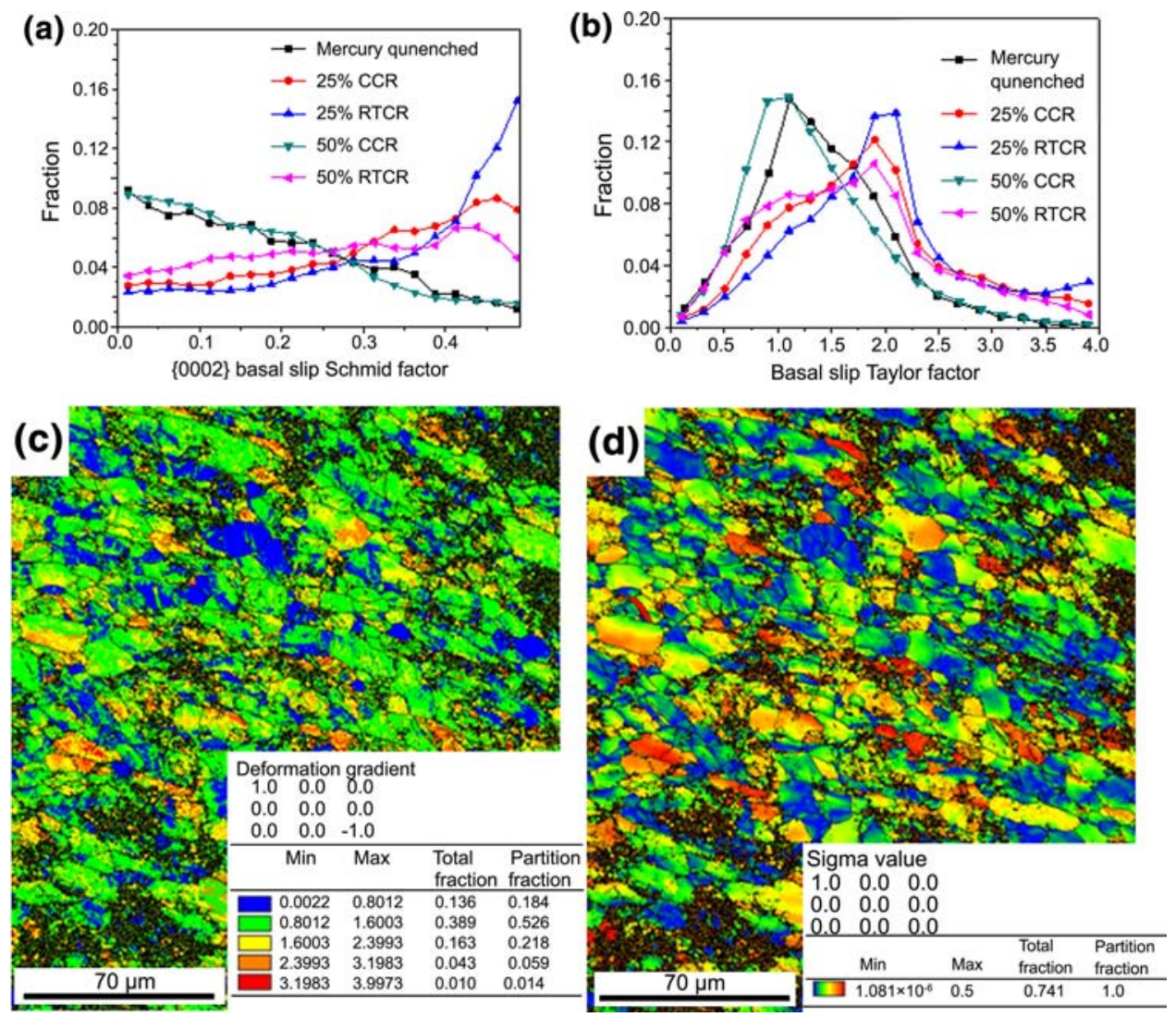

Fig. 9 Basal slip Taylor factor $\mathbf{a}$ and Schmid factor $\mathbf{b}$ values of CCR and RTCR zircaloy-2; basal slip Taylor factor image $\mathbf{c}$, Schmid factor image $\mathbf{d}$ of $50 \%$ CCR zircaloy- 2

are formed in zirconium [39]. If spacing between the dislocations with Burgers vector $b$ has a boundary $h$, then the crystal on either side of low-angle boundary is tilted by $\theta$

$\theta \approx 2 \sin \frac{\theta}{2}=\frac{b}{h}$,

This equation is also called Frank's formula [40]. When distance between the misorientation (step size) is $d$, area of the boundary planes with the neighboring points per unit volume can be represented by the length $l$ and area $A$ of hexagon, in consideration of unit thickness as

$\frac{(l / A)}{A}=\frac{\sqrt{3} d}{\sqrt{3} d^{2} / 2}=\frac{2}{d}$,

Then, the $\beta / h$ is the length of dislocation line per unit area, $\beta$ is a constant depends on geometry of dislocation arrangement. Value of $\beta$ is taken as 2 for tilt and 4 for twist boundaries. The dislocation line length per unit area and stored energy are given by $[18,31,41]$

$\rho \approx \frac{\beta \theta}{b d}$

$E=\rho \frac{G b^{2}}{2}$,

$E=\frac{\beta G b \theta}{2 d}$,

where $\theta$ is the average KAM between dislocation boundaries; $G$ is the shear modulus; $d$ is the step size.

The value of $\beta$ is taken as three due to deformed structure, mostly at low temperature in zircaloy-2. Since $\langle a\rangle$ slip is dominant, the value of $b$ corresponding to $\langle a\rangle$ slip is taken as $0.3232 \mathrm{~nm}$. Shear modulus of $34.8 \mathrm{GPa}$ for both CCR and RTCR samples is considered for comparison [42]. Step size for of EBSD scan is $0.1 \mu \mathrm{m}$. Table 4 shows

Table 4 Energy and dislocation density of cross rolled samples

\begin{tabular}{|c|c|c|c|c|c|}
\hline & Mercury quenched & $25 \% \mathrm{CCR}$ & $25 \%$ RTCR & $50 \% \mathrm{CCR}$ & $50 \%$ RTCR \\
\hline Average KAM (radian) & 0.008105 & 0.01951 & 0.017593 & 0.0276914 & 0.0233 \\
\hline Dislocation density $\rho\left(\mathrm{m}^{-2}\right)$ & & $9.944 \times 10^{14}$ & $8.805 \times 10^{14}$ & $1.818 \times 10^{15}$ & $1.41 \times 10^{15}$ \\
\hline Energy $(\mathrm{J} / \mathrm{mol})$ & & 29.02 & 25.70 & 53.06 & 41.15 \\
\hline
\end{tabular}


the value of dislocation density and energy of CCR and RTCR zircaloy-2. The variation in dislocation density and energy with increase in thickness reduction in RTCR and CCR shows that they are higher at cryo-temperature due to suppression of dynamic recovery. Although, above-mentioned method for calculation of energy gives more accurate results in case of medium and high stacking fault energy materials, but variation of stored energy after deformation can be compared in case of zircaloy-2 [31]. The formation of ultrafine grain during annealing is influenced by the stored strain energy also. The locations where the KAM is high, stored energy is also high, which on annealing recrystallization to form ultrafine grains.

\section{Conclusions}

Cross-rolling of zircaloy-2 at cryo-temperature leads to the formation of ultrafine grain structure in the bulk alloy even at $50 \%$ reduction. The tensile strength and ductility of cross-rolled zircaloy-2 are found to be $786 \mathrm{MPa}$ and $8.7 \%$, respectively. Annealing of deformed zircaloy-2 at $400{ }^{\circ} \mathrm{C}$ for $30 \mathrm{~min}$ leads to improvement in ductility of $9.8 \%$.

The deformation in CCR sample up to $25 \%$ reduction takes place by activation of prismatic, extension and contraction twinning, while further deformation up to $50 \%$ reduction occurs by primary contraction twinning and secondary extension twinning due to which basal texture become stronger and further deformation leads to failure. Activation of basal slip at cryo-temperature after 25\% rolling is also observed. Basal pole and EBSD image showed that at room temperature, deformation occurs by activation of various slip systems such as prismatic, pyramidal and basal at high strain. The CCR sample show larger stored energy $(53.06 \mathrm{~J} / \mathrm{mol})$ and dislocation density $\left(1.818 \times 10^{15} \mathrm{~m}^{-2}\right)$ as compared to RTCR due to suppression of dynamic recovery.

Acknowledgments One of the authors, Dr. R. Jayaganthan, expresses his sincere thanks to BRNS, Bombay for their financial grant to this work (No. BRN-577-MMD).

\section{References}

[1] S.R. Macewen, J. Faber Jr., A.P.L. Turner, Acta Mater. 31, 657 (1983)

[2] R.L. Mehan, F.W. Wiesinger, Mechanical Properties of Zircaloy-2, AEC Research and Development Report (1961)

[3] E.F. Ibrahim, In-Reactor Creep of Zirconium-Alloy Tubes and Its Correlation with Uniaxial Data: Applications-Related Phenomena for Zirconium and Its Alloys, ASTM STP 458, American Society for Testing and Materials (1969), pp. 18-36

[4] R.G. Ballinger, R.M. Pelloux, J. Nucl. Mater. 97, 231 (1981)
[5] K. Linga Murty, I. Charit, Prog. Nucl. Energy 48, 325 (2006)

[6] E. Tenckhoff, Deformation Mechanisms, Texture, and Anisotropy in Zirconium and Zircaloy, American Society for Testing and Materials (1988)

[7] Y.N. Wang, J.C. Huang, Mater. Chem. Phys. 81, 11 (2003)

[8] R.J. McCabe, E.K. Cerreta, A. Misra, G.C. Kaschner, C.N. Tome, Philos. Mag. A 86, 3595 (2006)

[9] M. Knezevic, I.J. Beyerlein, T. Nizolek, N.A. Mara, Mater. Res. Lett. 1, 133 (2013)

[10] R.J. McCabe, G. Proust, E.K. Cerreta, A. Misra, Int. J. Plast 25, 454 (2009)

[11] A. Akhtar, Metall. Trans. A 6(1217), 1217-1222 (1975)

[12] G. Monnet, B. Devincre, L.P. Kubin, Acta Mater. 52, 4317 (2004)

[13] G.W. Groves, A. Kelly, Philos. Mag. 89, 877 (1963)

[14] A. Akhtar, Acta Metall. 21, 1 (1973)

[15] C.N. Tome, P.J. Maudlin, R.A. Lebensohn, G.C. Kaschner, Acta Mater. 49, 3085 (2001)

[16] A. Akhtar, J. Nucl. Mater. 47, 79 (1973)

[17] B.F. Luan, Q. Ye, J.W. Chen, H.B. Yu, D.L. Zhou, Y.C. Xin, Trans. Nonferrous Met. Soc. China 23, 2890 (2013)

[18] Y. Takayama, J.A. Szpunar, Mater. Trans. 45, 2316 (2004)

[19] C.S. Barrett, T.B. Massalski, Structure of Metals (McGraw-Hill Book Company, New York, 1966)

[20] S. Goel, N. Keskar, R. Jayaganthan, I.V. Singh, D. Srivastava, G.K. Dey, N. Saibaba, Mater. Sci. Eng. A 603, 23 (2014)

[21] S. Goel, R. Jayaganthan, I.V. Singh, D. Srivastava, G.K. Dey, N. Saibaba, Mater. Des. 55, 612 (2014)

[22] H.L. Cox, D.G. Sopwith, Proc. Phys. Soc. 49, 134 (1937)

[23] H.J. Bunge, Kristall Technik 5, 145 (1970)

[24] J.H. Shen, Y.L. Li, Q. Wei, Mater. Sci. Eng. A 582, 270 (2013)

[25] W.B. Hutchinson, Int. Met. Rev. 29, 25 (1984)

[26] M. Matsuo, S. Hayami, S. Nagashima, Adv. X-Ray Anal. 14, 214 (1971)

[27] B.L. Averbach, M.B. Bever, M.F. Comerford, J.S. Leach, Acta Metall. 4, 477 (1956)

[28] R.L. Every, M. Hartherly, Texture 1, 183 (1974)

[29] J.S. Kalland, Y.C. Huang, Met. Sci. 18, 381 (1984)

[30] M. Taheri, H. Weiland, A. Rollett, Metall. Mater. Trans. A 37, 19 (2006)

[31] A. Godfrey, W.Q. Cao, Q. Liu, N. Hansen, Metall. Mater. Trans. A 36, 2371 (2005)

[32] N.P. Gurao, S. Sethuraman, S. Suwas, Metall. Mater. Trans. A 44, 1497 (2013)

[33] D.F. Guo, M. Li, Y.D. Shi, Z.B. Zhang, H.T. Zhang, X.N. Liu, B.N. Wei, X.Y. Zhang, Mater. Des. 34, 275 (2012)

[34] D.F. Guo, M. Li, Y.D. Shi, Z.B. Zhang, T.Y. Ma, H.T. Zhang, X.Y. Zhang, Mater. Sci. Eng. A 558, 611 (2012)

[35] S.K. Sahoo, V.D. Hiwarkar, I. Samajdar, G.K. Dey, D. Srivastav, R. Tiwari, S. Banerjee, Scr. Mater. 56, 963 (2007)

[36] C.D. Judge, Thesis, Queen's University Kingston (2009)

[37] Q. Yu, J. Sun, J.W. Morris Jr, A.M. Minor, Scr. Mater. 69, 57 (2013)

[38] C.H. Cáceresa, P. Lukáč, Philos. Mag. 88, 977 (2008)

[39] G. Monnet, B. Devincre, L.P. Kubin, Acta Mater. 52, 4317 (2004)

[40] F.C. Frank, Report on the Symposium on the Plastic Deformation of Crystalline Solids (Carnegie Institute of Technology, Carnegie, 1950), pp. 150-154

[41] Q. Liu, D.J. Jensen, N. Hansen, Acta Mater. 46, 5819 (1998)

[42] T. Unga, O. Castelnau, G. Ribarik, M. Drakopoulos, J.L. Bechade, T. Chauveau, A. Snigirev, I. Snigireva, C. Schroer, B. Bacroix, Acta Mater. 55, 1117 (2007) 